\title{
Localizations of $\gamma$-Actins in Skin, Hair, Vibrissa, Arrector Pili Muscle and Other Hair Appendages of Developing Rats
}

\author{
Kiyokazu Morioka ${ }^{1,2}$ and Hiromi Takano-Ohmuro ${ }^{1}$ \\ ${ }^{1}$ Research Institute of Pharmaceutical Sciences, Musashino University, Nishitokyo City, Tokyo, Japan and ${ }^{2}$ The Tokyo \\ Metropolitan Institute of Medical Science, Tokyo, Japan*
}

Received October 13, 2015; accepted February 16, 2016; published online April 9, 2016

\begin{abstract}
Six isoforms of actins encoded by different genes have been identified in mammals including $\alpha$-cardiac, $\alpha$-skeletal, $\alpha$-smooth muscle ( $\alpha$-SMA), $\beta$-cytoplasmic, $\gamma$-smooth muscle ( $\gamma$-SMA), and $\gamma$-cytoplasmic actins ( $\gamma-C Y A)$. In a previous study we showed the localization of $\alpha$-SMA and other cytoskeletal proteins in the hairs and their appendages of developing rats (Morioka K., et al. (2011) Acta Histochem. Cytochem. 44, 141-153), and herein we determined the localization of $\mathrm{Y}$ type actins in the same tissues and organs by immunohistochemical staining. Our results indicate that the expression of $\mathrm{y}$-SMA and $\mathrm{y}-\mathrm{CYA}$ is suggested to be poor in actively proliferating tissues such as the basal layer of the epidermis and the hair matrix in the hair bulb, and as well as in highly keratinized tissues such as the hair cortex and hair cuticle. In contrast, the expression of $y$-actins were high in the spinous layer, granular layer, hair shaft, and inner root sheath, during their active differentiations. In particular, the localization of $y$-SMA was very similar to that of a-SMA. It was located not only in the arrector pili muscles and muscles in the dermis, but also in the dermal sheath and in a limited area of the outer root sheath in both the hair and vibrissal follicles. The Y-CYA was suggested to be co-localized with $\mathrm{y}$-SMA in the dermal sheath, outer root sheath, and arrector pili muscles. Sparsely distributed dermal cells expressed both types of $\mathrm{y}$-actin. The expression of $\mathrm{y}$-actins is suggested to undergo dynamic changes according to the proliferation and differentiation of the skin and hair-related cells.
\end{abstract}

Key words: gamma actin, hair, skin, arrector pili muscle, differentiation

\section{Introduction}

Actins and their ancestor proteins are known to be ubiquitously distributed in prokaryotic and eukaryotic cells $[6,11]$. They play a central role in cell movement, maintenance of cell shape, muscle contraction, and many other biological processes [24, 29, 32, 33]. In mammals, six dif-

Correspondence to: Hiromi Takano-Ohmuro, Ph. D., Research Institute of Pharmaceutical Sciences, Musashino University, 1-1-20 Shinmachi, Nishitokyo-City, Tokyo 202-8585, Japan.

E-mail: h_ohmuro@musashino-u.ac.jp

*The Tokyo Metropolitan Institute of Medical Science was reorganized after the transfer of K.M. and the present name is Tokyo Metropolitan Institute of Medical Science. ferent actin isoforms encoded by separate genes have been identified $[18,27,37]$. The six isoforms include, $\alpha$-skeletal muscle $(\alpha$-SKA), $\alpha$-cardiac muscle ( $\alpha$-CAA), $\alpha$-smooth muscle ( $\alpha$-SMA), $\beta$-cytoplasmic ( $\beta$-CYA), $\gamma$-smooth muscle $(\gamma$-SMA) and $\gamma$-cytoplasmic ( $\gamma$-CYA) actins. The names of these isoforms do not exactly mean their functions. For example, $\gamma$-CYA is known to be a Z-disc component in myofibers of the skeletal muscle $[25,26]$. These actin isoproteins were suggested to be functionally specified for the tissues in which they predominate $[12,23,28,36]$. The most powerful tool to investigate the roles of protein isoforms has been the gene modified mouse. However, the investigators may be confronted with the difficult problem that several isoforms may, at least partially, compensate the depletion of other isoforms $[15,35]$. Therefore the interpretation of knock-out mouse model experiments has fre- 
quently been complicated. Furthermore actin cables may be formed by discretionary combination of various isoforms, and accordingly they are possible to have numerous properties like alloy [27]. Considering these potential problems, we believe that the localization studies of actins by immunohistochemistry are still needed to investigate the contribution of each isoform.

A quarter of a century ago, McHugh et al. [17] carried out a comprehensive analysis of the expression of the isoactin multigene family in the rat, although the skin, hair and their appendages were not included in their list. According to their results, $\gamma$-SMA expression was observed mainly in the intestine before birth, but was mainly expressed in the bladder and the stomach after birth. As for $\gamma$-CYA, they reported that the expression was high in the lung and the intestine before birth, and was high in the lung and other various organs after birth. Thereafter, some experimental results regarding the functions of $\gamma$-actins were published. Studies investigating pathogenic mutations in humans revealed that a $\gamma$-SMA mutation causes deafness [38]. The distinct intracellular localization of $\beta$ and $\gamma$-CYA in a cultured cell suggested that each cytoplasmic actin presented its own role; $\beta$-actin is involved in producing unbranched filamentous arrays in stress fibers, while $\gamma$-actin produces branched meshwork in cortical and lamellar structures [4]. $\gamma$-CYA expression was reported to be elevated about 10 fold in the muscle of dystrophin-deficient mice [8]. $\gamma$-CYA null mice were found to be fully viable before birth, but most died within 48 hours after birth due to respiratory failure and cannibalization by the parents [3]. At present, the roles of $\gamma$-actins, in particular those of $\gamma$-SMA still remain unclear.

We previously investigated the distribution of actins and myosins in the hair follicle, its appendages, and other skin tissues of rats from fetal to adult stages as a base to elucidate their roles [22]. The results showed that nonmuscle myosin and actin co-localize in the dermal sheath and outer root sheath of the hair follicles as well as in the spinous and granular layers of the skin. We then studied the rise and decay of $\alpha$-SMA expression in the arrector pili muscle, hair follicle, and other skin tissues [21]. Our conclusions were that, 1) a strong $\alpha$-SMA signal in the skin was observed in the lower part of the dermal sheath and outer root sheath in both the hair and vibrissal follicles from fetal to adult stages, 2) vimentin was expressed over the entire height of the dermal sheath, and 3) the strongest $\alpha$-SMA signal in the arrector pili muscle was observed at 1-2 weeks after birth. Based on these results, we speculated that the presence of actin-myosin fibers in dermal sheath and outer root sheath is beneficial for the hair follicle to cope with the movement of the hair shaft caused by physical contacts with outside materials or by the contraction of internal muscles. Besides $\alpha$-SMA, another type of smooth muscle actin, "enteric" $\gamma$-SMA is known [18], although the information about this protein is poor. Recently Arnoldi et $a l$. presented a new theory that $\alpha$-SMA is responsible for the contractile activity and $\gamma$-SMA is involved in the compliance of smooth muscle and related cells [1]. This theory aroused our interest regarding the localization of $\gamma$-SMA in the hair and skin.

It has been an enigma for a long time that what is the specific role of $\gamma$-SMA that is not compensatable by $\alpha$ SMA and why the DNA sequence of $\gamma$-SMA gene has been conserved during the long history of vertebrates. Recently Klar et al. made a breakthrough. They reported that mutation of the $\gamma$-SMA gene may cause familial visceral myopathy and parturition difficulties due to weak uterine contractions [14]. It may explain one of the reasons of the long conservation of $\gamma$-SMA gene in mammals. $\gamma$-CYA and $\gamma$-SMA present an almost identical isoelectric point and are both classified into class I isoactin. However, they play different roles $[12,27]$. The amino-acid sequences of $\gamma$-SMA in rat, mouse, and human are known to be identical, and they are the same for $\gamma$-CYA in those mammalian species, suggesting that the functions of these proteins may be conserved in most mammals during their evolution. Herein, we investigated the localization of $\gamma$-SMA and $\gamma$-CYA in the arrector pili muscle, hair follicle, vibrissa and other skin tissues, and discussed their possible roles.

\section{Materials and Methods}

Fourteen-day pregnant Sprague Dawley rats were obtained from Nippon SLC Inc., Hamamatsu, Japan. Treatment of animals was carried out according to the guidelines of the Tokyo Metropolitan Institute of Medical Science, which conform to the principles of laboratory animal care of the NIH. The appearance of the vaginal plug was used to indicate day 0 of gestation. Delivery of newborn animals was observed at gestational day 21 or 22 . In this study, day 0 represents the day of birth. Several paraffin blocks obtained from different littermate groups were used for one series of experiments. The preparation of samples, and the methods of immunohistochemistry were performed as described before [21]. In brief, dorsal skins and mystacial pads of fetal, neonatal and adult animals were fixed in $10 \%$ formalin neutral buffer solution (Wako Pure Chemical Industries Ltd., Osaka, Japan) for several days at $4^{\circ} \mathrm{C}$, dehydrated, and embedded in paraffin. The paraffin blocks were sectioned at $6-\mu \mathrm{m}$ thickness for immunohistochemistry. A monoclonal murine antibody (clone 2A3) against $\gamma$ CYA (ABIN1723318) and a polyclonal rabbit antibody against $\gamma$-SMA (ABIN483824) were obtained from antibodies-online $\mathrm{GmbH}$. Although these antibodies were produced using human antigens, the sequences of $\gamma$-CYA and $\gamma$-SMA are identical between human and rat proteins (http://www.uniprot.org/), respectively, ensuring the specificity of the antibodies that proposed by the manufacturer in the product manual. In control experiments, the treatment of primary antibody was omitted. The specimens were dehydrated and treated with 3\% hydrogen peroxide for 60 min, followed by washing 3 times with phosphate buffered 


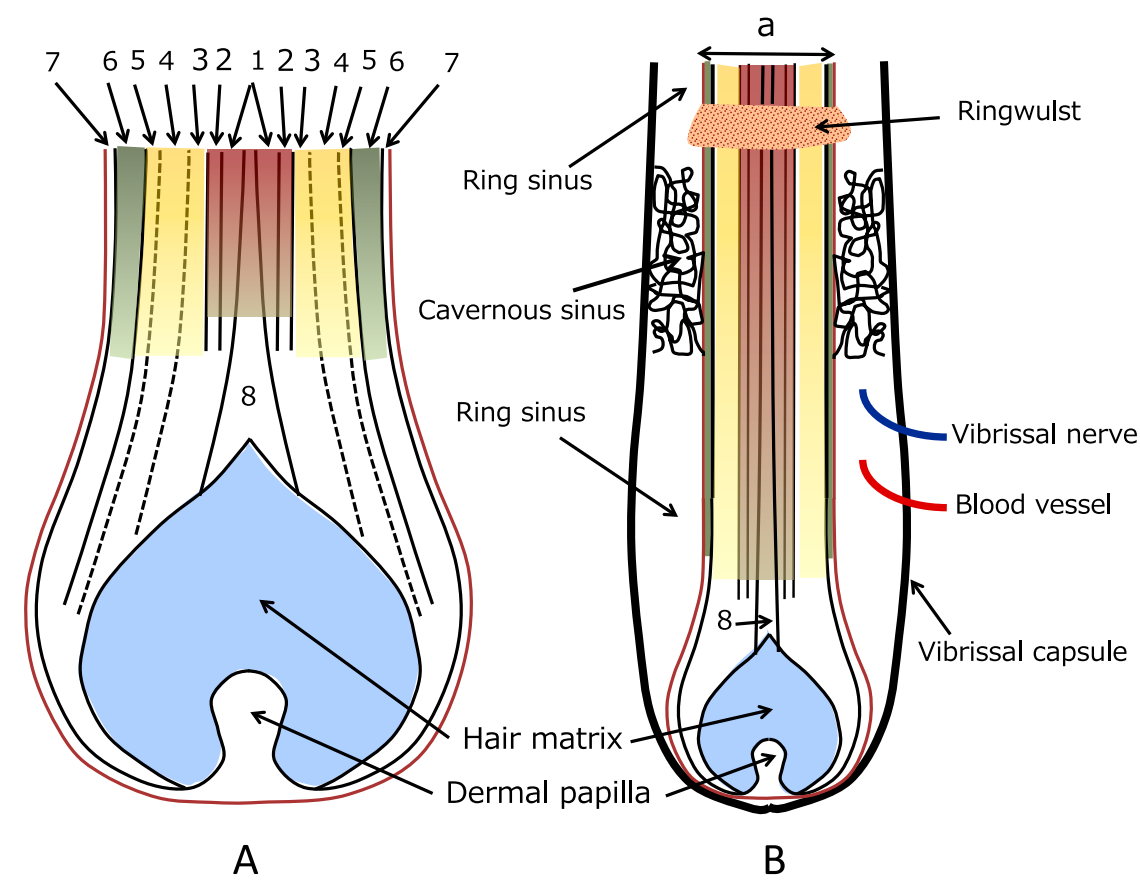

Fig. 1. Schematic representation of hair (A) and vibrissal (B) follicles. The vibrissal follicle consists of a pelage-type hair follicle designated by area a shown in $\mathbf{B}$ and a surrounding sinus encapsulated by the vibrissal capsule. The parts of in the area a are essentially the same with those in the pelagetype hair follicle shown in A. The sinus in the vibrissal capsule contains ring sinus, cavernous sinus, interpenetrated nerves and blood vessels, ringwulst etc. The names of the parts in the hair follicle are, 1: hair cortex, 2: hair cuticle, 3: cuticle of inner root sheath, 4: Huxley's layer of inner root sheath, 5: Henle's layer of inner root sheath, 6: outer root sheath, 7: dermal sheath, and 8: hair medulla.

saline pH 7.2-7.4 (Wako, Osaka, Japan), and incubated with $10 \%$ goat serum (Nichirei Co. Tokyo, Japan) for 60 min at room temperature to block nonspecific staining. They were then washed 3 times with the phosphate buffered saline again. The incubation of the sections with primary antibodies was carried out for $24 \mathrm{hr}$ at $4^{\circ} \mathrm{C}$. After incubation, sections were washed 4 times with phosphate buffered saline and incubated with a secondary antibody for $60 \mathrm{~min}$ at room temperature. We used Histofine simplestain rat MAX-PO (MULTI) as the secondary antibody, which was obtained from Nichirei Co. In order to circumvent the autofluorescence of epidermal tissues and substances derived from erythrocytes, the detection of secondary antibody was not carried out by immunofluorescence. The proteins were visualized using 3-3'-diaminobenzidine (DAB). The sections stained by DAB were briefly poststained with methylgreen-pyronine [22]. An Olympus BX-51 microscope equipped with an Olympus E-420 camera system was used to obtain photographs.

\section{Results}

\section{Structures of hair and vibrissa}

Schematic representations of the hair follicle (A) and the vibrissal follicle (B) are shown in Fig. 1. The tissues numbered $1-6,8$ and hair matrix belong to the epidermis, while 7 and dermal papilla are included in the dermis. Some hair follicles lack medulla (8 in Fig. 1A). Hair shaft cells $(1,2$ in Fig. 1A) and inner root sheath cells (3-5 in
Fig. 1A) are formed by the proliferation and differentiation of the hair matrix cells. The outer root sheath (6 in Fig. 1A) consists of several types of cells. The basement membrane (an intercellular space) is observed between the outer root sheath and the dermal sheath. In the vibrissal follicle, the ordinary hair follicle components shown Fig. 1A are wrapped by the vibrissal capsule that forms a robust bag filled with blood (Fig. 1B). The cavernous sinus and ringwulst stabilize the hair in the blood and may play a role as cushions also. The movement of the vibrissa is perceived by the sensory nerve introduced into the bag. The whole bag can be incurved by striated muscles attached to the outside surface of the bag voluntarily. In contrast, the hair follicle is incurved by the arrector pili muscle involuntarily. The abbreviations are shown in the Section V.

\section{Dorsal skin on day 16 of gestation}

At day 16 of gestation, the skin of the fetal rat was covered by an outermost cellular monolayer, periderm. The fetal epidermis adjoins the inner side of the periderm. In the fetal epidermis on day 16 of gestation, the four discrete layers (basal, spinous, granular, and cornified) characteristic of the adult epidermis had not yet appeared [16, 20]. The staining of the cornified layer after birth may fluctuate due to the possible contaminations from maternal or outer world substances. Therefore, the staining of the skin surface of the animals before birth is expected to be more reliable. As shown in Fig. 2G and Fig. 3I, non-specific irregular staining of the skin surface was not observed in 


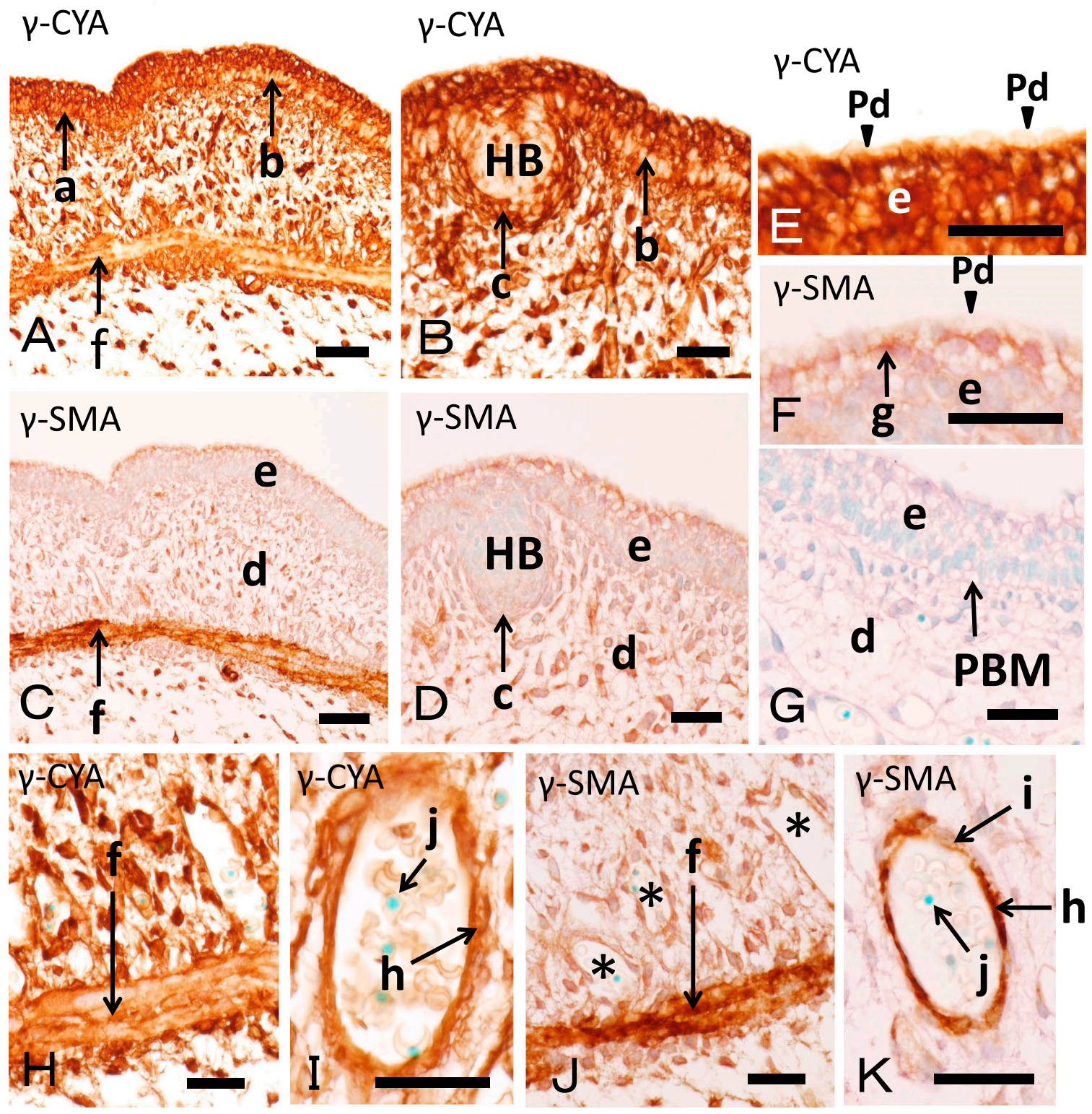

Fig. 2. Immunohistochemical staining for $\gamma$-CYA $(\mathbf{A}, \mathbf{B}, \mathbf{E}, \mathbf{H}, \mathbf{I})$ and $\gamma$-SMA $(\mathbf{C}, \mathbf{D}, \mathbf{F}, \mathbf{J}, \mathbf{K})$ in the dorsal skin of fetal rats on day 16 of gestation. The result of a control experiment, in which the treatment of primary antibody was omitted, is shown in $\mathbf{G}$. The control experiments shown in following figures were carried out in the same way. The target protein in this and the following figures to be detected by its antibody is shown in each panel, when different targets are shown in the fame figure. See Section V. for the abbreviations. Symbols are a: the innermost cellular layer of the fetal epidermis, b: unstained cells in the innermost cellular layer of the fetal epidermis, c: cells that surround the hair bud (HB), d: dermis, e: fetal epidermis, f; presumptive dermal muscle, g: outermost part of the fetal epidermis, h: blood vessel wall in the dermis, i: unstained area in the vessel wall, and j: nucleated fetal erythrocyte. Asterisks designate the blood vessels. Bars $=50 \mu \mathrm{m}(\mathbf{A}, \mathbf{C})$ and $25 \mu \mathrm{m}$ (other panels).

the control experiment at stages before birth, whereas a control experiment carried out on day 2 after birth shows a slight non-specific staining in the skin surface (Fig. 5F). On day 16 of gestation, $\gamma$-CYA was suggested to be expressed in most parts of the skin (Fig. 2A) except in the basal part of the epidermis and the hair bud (arrow $b$ and HB, respectively in Fig. 2B). Detailed observation revealed stained (arrow a in Fig. 2A) and unstained (arrow b in Fig. 2A) areas in the basal part of the epidermis. Since the epidermis undergoes a rapid differentiation around this stage, this uneven staining pattern may depend on the local difference in progress of the differentiation. In subsequent studies, the basal layer of the skin was found to be stained for $\gamma$-CYA at an invariant low level in contrast to the upper layers on day 19 of gestation as shown in Fig. 3A. It suggests that the stained area in Fig. 2A may still be in the earlier stage of the differentiation.

The hair bud cells derived from the basal layer of the epidermis expressed $\gamma$-CYA at a low level (HB in Fig. 2B). In contrast, the dermal cells surrounding the hair bud (arrow c in Fig. 2B), as well as the sparsely distributed fibroblasts in the dermis, expressed high levels of $\gamma$-CYA. 
The blood vessel wall was also stained positive for $\gamma$-CYA, while fetal erythrocytes were not (Fig. 2I). In the presumptive dermal muscle, the staining for $\gamma$-CYA was light in comparison to the surrounding fibroblasts (arrows $\mathrm{f}$ in Fig. $2 \mathrm{~A}$ and $2 \mathrm{H}$ ). Fig. $2 \mathrm{H}$ represents the staining of the cells in area $\mathrm{f}$ is conspicuous only at the periphery of the cells. The periderm was not stained for $\gamma$-CYA (arrowheads Pd in Fig. 2E). The periderm consists of a monolayer of cells located at the surface of the whole embryo and functions before the formation of the keratinized epidermis [30]. Fig. 2E also shows that the middle and outer (near periderm) area of the primitive epidermis (area "e") consists of a random mixture of deeply and lightly stained cells, suggesting the heterogenous or an undirected state of the tissue on day 16 of gestation.

In contrast to the extensive expression of $\gamma$-CYA, the prominent expression of $\gamma$-SMA was restricted in the presumptive dermal muscle (arrows $\mathrm{f}$ in Fig. $2 \mathrm{C}$ and $2 \mathrm{~J}$ ) and in vascular cells (arrow h in Fig. 2K). As shown in Fig. 2J, the staining of area $\mathrm{f}$ is deep in comparison to the surrounding fibroblasts. Although the wall of blood vessel in Fig. 2J (asterisks) were not stained deeply, some vessel walls were found to contain both $\gamma$-SMA-stained (arrow h in Fig. 2K) and unstained (arrow $\mathrm{i}$ in Fig. 2K) areas. Whether this heterogeneity depends on the stages of differentiation of the vascular cells or not is undetermined. The most sparsely distributed dermal fibroblasts were moderately stained for $\gamma$-SMA, but some of them seemed to be negative for the staining (near-epidermal part of the area d in Fig. 2D). The cells surrounding the hair follicles were negative in the staining (arrows $\mathrm{c}$ in Fig. 2D). On day 16 of gestation, $\gamma$ SMA staining in epidermal cells (e in Fig. 2C, 2D and 2F) was generally negative except for the outermost epidermal cellular layer that lies just beneath the periderm (arrow $g$ in Fig. 2F), which was moderately positive for $\gamma$-SMA. Since the results regarding the localization of $\gamma$-CYA suggested a random mixture of heterogeneous cells, this result was a surprise (e in Fig. 2E). Furthermore, the staining for $\gamma$-SMA appeared to be restricted to the intracellular area which is facing the periderm (Fig. 2F), suggesting that the $\gamma$-SMA may contribute to make the cell polarity. The adult-like four (cornified, granular, spinous and basal) layers are not built in the primitive epidermis, but some undetermined layer structures may already be formed on day 16 of gestation. The observations by electron microscopy, indicating a specific morphology of the cells facing the periderm, also support this idea [20]. The staining by methylgreen-pyronine (control experiment: Fig. 2G) showed a distinguishable light-green-stained layer just above the presumptive basement membrane (PBM), but other cells in "e" do not display such a color. The periderm did not show the signal of both $\gamma$-SMA (Fig. 2F) and $\gamma$-CYA (Fig. 2E). On day 16 of gestation, some nucleated erythrocytes were observed as designated by arrows $\mathrm{j}$ in Fig. $2 \mathrm{I}$ and $2 \mathrm{~K}$ (nuclei were stained by methylgreen-pyronine). The erythrocytes including these nucleated ones showed essentially no signal of $\gamma$-SMA (Fig. 1K) and $\gamma$-CYA (Fig. 2I). This result is consistent with a previous result indicating that the major actin in erythrocytes is $\beta$-actin [28].

\section{Dorsal skin on day 19 of gestation}

From day 16 to day 19 of gestation, the epidermis became thick and transformed into the adult-like structure consisting of cornified, granular, spinous, and basal layers (Fig. 3A, 3B, 3D, 3E, and ref. 20). The periderm disappears during this period. In addition to the hair bud, primitive hair follicles and those accompanied by the dermal papilla were observed (Fig. 3A, 3B and Fig. 4D, 4G). As the differentiation proceeds, the contrast of the staining for $\gamma$-CYA between positive areas including the spinous and the granular layers (designated by a in Fig. 3A and 3B) and the negative basal layer (designated by $\mathrm{b}$ in Fig. $3 \mathrm{~A}$ and $3 \mathrm{~B}$ ) of the epidermis became conspicuous. In particular, the very deep staining of the granular layer was evident (Fig. 3A, 3B, and 4C) on day 19 of gestation. Dermal cells that surround the hair bud (a in Fig. 4A), sparsely distributed dermal cells (Fig. 3A, 3B, and 4A-C), dermal sheath cells that surround the hair follicle (DS in Fig. 3A, 3B, 4B, and 4C), and dermal papilla cells (DP in Fig. 3A, 3B, and 4D) were positive in the staining for $\gamma$-CYA. Epidermal cells in the hair buds as well as those in the primitive hair follicles were negative or slightly positive in the staining for $\gamma$-CYA (HB in Fig. 4A and PHF in Fig. 4B-D).

Similar to $\gamma$-CYA, $\gamma$-SMA was suggested to express in the spinous, granular, and cornified epidermal layers on day 19 of gestation (Fig. 4E and 4F). The staining was almost undetectable in the dermal sheath and dermal papilla (Fig. 3D, 3E, 4E-G). Sparsely distributed dermal cells were recognizable to be positive in for $\gamma$-SMA (Fig. 4E-G) when compared with the control experiment (Fig. 4H). The epidermis just above some hair follicles was not stained for $\gamma$ CYA or $\gamma$-SMA from the basal to the near-surface area (designated by $\mathrm{c}$ in Fig. 4D and 4F). We speculate that the basal layer may expand in this area to form the hair follicle.

\section{Mystacial pad on day 19 of gestation}

The staining patterns of $\gamma$-actins in the mystacial pad were approximately the same as that observed in the dorsal skin, except for the following two points. First, the muscle cells are some of the major components of the dermis in the mystacial pad. One of this cell type is designated by arrow $\mathrm{f}$ in Fig. 3F and is positive for $\gamma$-SMA, and is also positive for $\gamma$-CYA (Fig. 3C), $\alpha$-SKA [22], and $\alpha$-SMA [21]. The sections in Fig. 3C and Fig. 3F are adjacent. These multiple-actin positive cells may be precursors of the striated muscles which will be formed after birth (Fig. 9J-L), though they were observed sporadically at the stage on day 19 of gestation. The striated muscles are known to be abundant in the dermis of the mystacial pad of adult animals [7]. The other characteristic of the mystacial pad on day 19 is the very thick cellular layers that surround vibrissal follicles. Some vibrissae are still in the stage of the bud (Fig. 

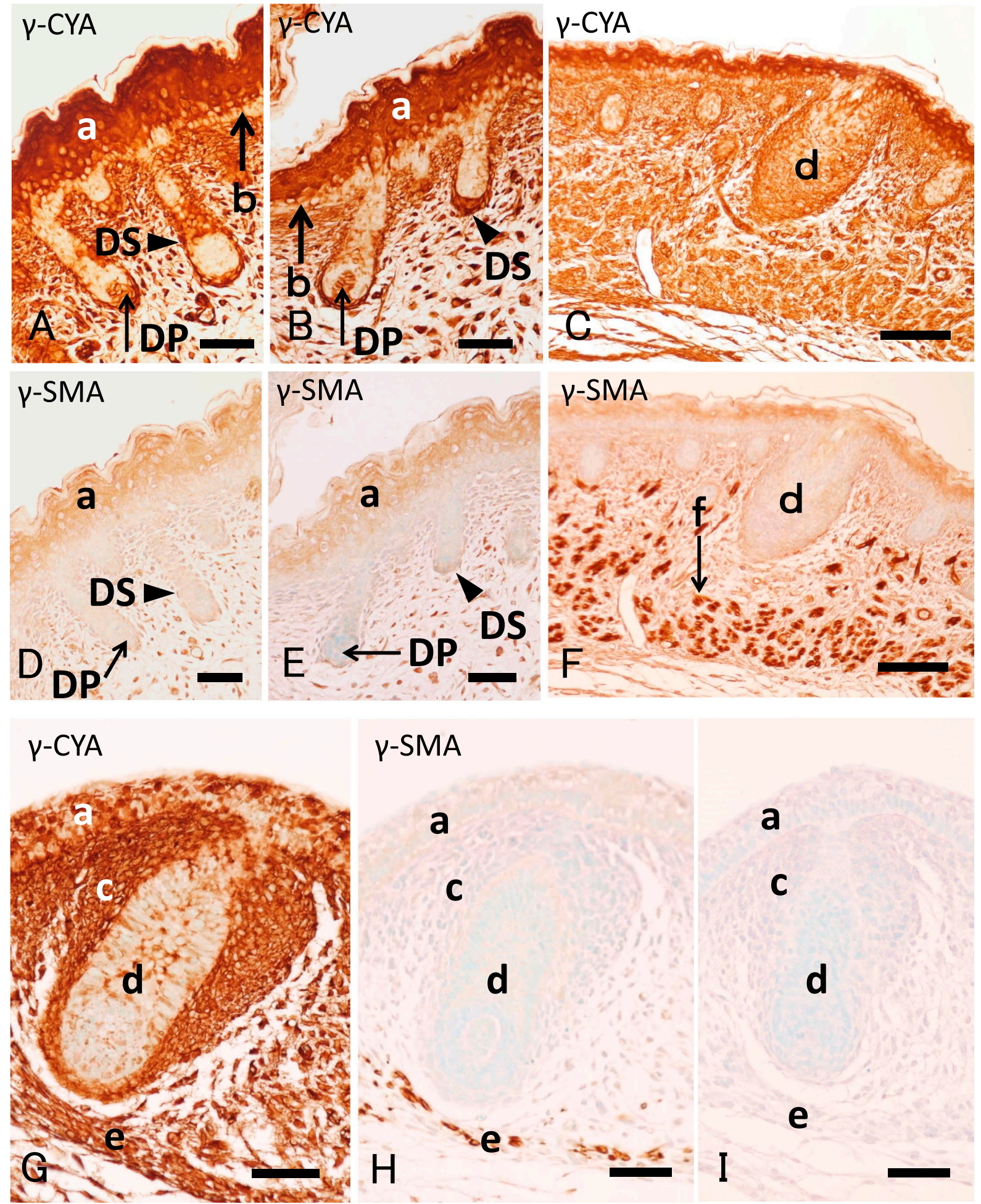

Y-SMA

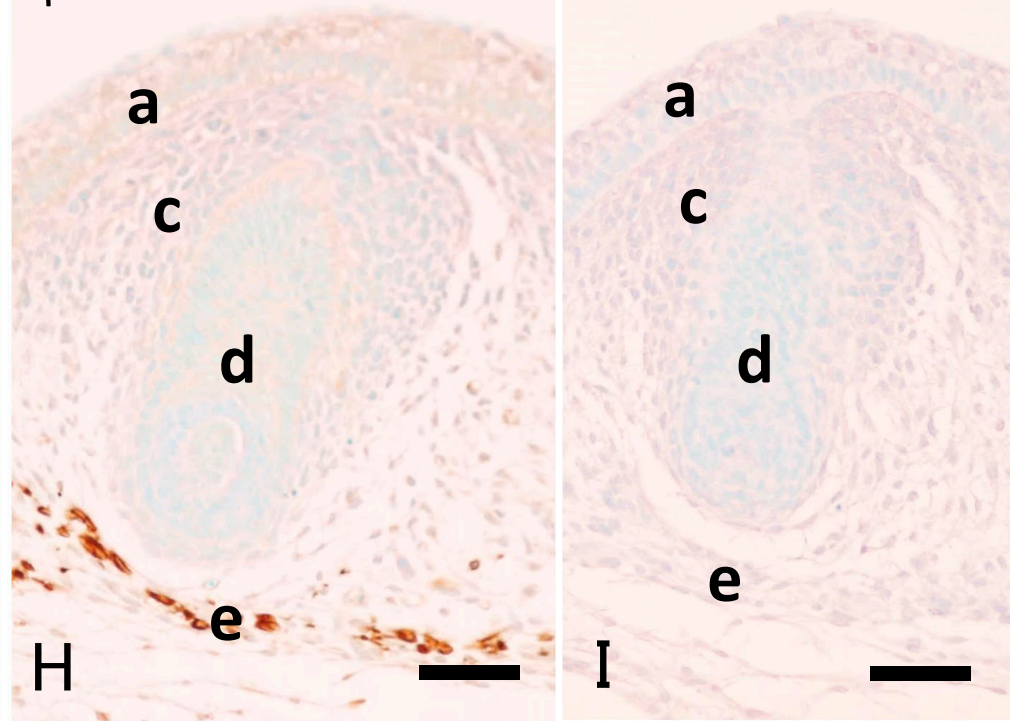

Fig. 3. Immunohistochemical staining for $\gamma$-CYA $(\mathbf{A}-\mathbf{C}, \mathbf{G})$ and $\gamma$-SMA $(\mathbf{D}-\mathbf{F}, \mathbf{H})$ in the dorsal skin $(\mathbf{A}, \mathbf{B}, \mathbf{D}, \mathbf{E})$ or in the mystacial pad $(\mathbf{C}, \mathbf{F}, \mathbf{G}-\mathbf{I})$ of fetal rats on day 19 of gestation. The result of a control experiment is shown in I. Symbols are a: spinous and granular layers of the epidermis, b: basal layer of the epidermis, c: dermal cells that surround the vibrissal bud, d: vibrissal bud, e: muscle cell mass, f: presumptive dermal muscle cells in the mystacial pad. Bars $=50 \mu \mathrm{m}(\mathbf{A}, \mathbf{B}, \mathbf{D}, \mathbf{E}, \mathbf{G}-\mathbf{I})$ and $100 \mu \mathrm{m}(\mathbf{C}, \mathbf{F})$.

3G), while others grew to form primitive vibrissal follicles (Fig. 4I and 4J). In both developmental stages, the surrounding cell mass including presumptive vibrissal capsule cells, presumptive sinus cells, dermal sheath cells and other precursor cells are shown to be entirely positive in the staining for $\gamma$-CYA (c in Fig. 3G, g in Fig. 4I and 4J). In contrast to the surrounding cell mass, the percentage of the $\gamma$-CYA-positive cells was small in the main body of the 


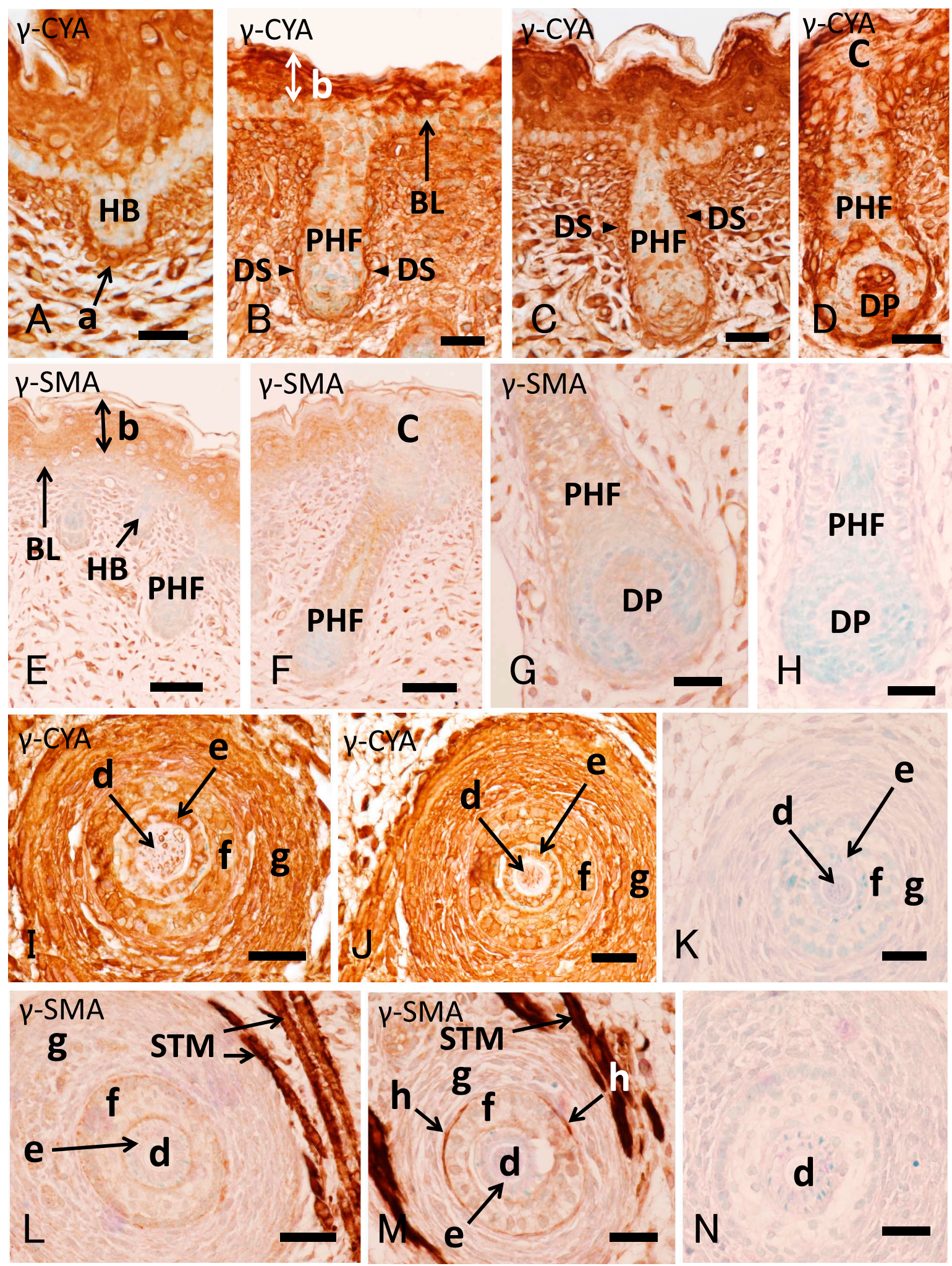

Fig. 4. Immunohistochemical staining for $\gamma$-CYA $(\mathbf{A}-\mathbf{D}, \mathbf{I}, \mathbf{J})$ and $\gamma$-SMA $(\mathbf{E}-\mathbf{G}, \mathbf{L}, \mathbf{M})$ on day 19 of gestation. Views of primitive hair follicles $(\mathbf{A}-\mathbf{H})$ and primitive vibrissae $(\mathbf{I}-\mathbf{N})$. Results of control experiments are shown in $\mathbf{H}, \mathbf{K}, \mathbf{N}$. Symbols are a: cells that surround hair bud, b: epidermis with the exception of the basal layer, c: unstained region in the epidermis, d: vibrissal shaft, e: inner root sheath, f: outer root sheath, g: dermal cells surrounding the vibrissal follicle, h: special areas positive for (probably dermal sheath) positive for $\gamma$-SMA. BL and PHF means basal layer and primitive hair follicle, respectively. See Section V. for the abbreviations. Bars=25 $\mu \mathrm{m}$ (except $\mathbf{E})$ and $50 \mu \mathrm{m}(\mathbf{E})$. 
vibrissal bud (d in Fig. 3G). However, when it grew into the vibrissal follicle, inner and outer root sheathes were positive in the staining for $\gamma$-CYA (e and $\mathrm{f}$ areas in Fig. 4I and $4 \mathrm{~J}$ ). The staining for $\gamma$-SMA in the adjacent section of Fig. $3 \mathrm{G}$ was evident only in the deep area of the dermis beneath the primitive vibrissa (e in Fig. $3 \mathrm{H}$ ), but when it grew into the differentiated vibrissa, the staining for $\gamma$ SMA was widely seen in the muscle cells surrounding vibrissal follicles (STM in Fig. 4L and 4M). The stage of differentiation was uneven from one area to the other in the mystacial pad on day 19 of gestation. In the differentiated vibrissa on day 19 of gestation, the boundary area between the outer root sheath and the capsule, probably it corresponds to dermal sheath, was stained (arrow h in Fig. 4M). This staining was not as clear in the section shown in Fig. $4 \mathrm{~L}$ that corresponds to the lower position than that of Fig. 4M. Generally, the $\gamma$-CYA staining of hair shaft was weak (d in Fig. 4I and 4J), and $\gamma$-SMA staining was negative (d in Fig. 4L and 4M).

\section{Dorsal skin during the neonatal period}

Our previous results showed that $\alpha$-SMA expression in the arrector pili muscle of the rat had been mostly observed during the neonatal period [21]. The present experiment shows that $\gamma$-SMA became detectable in the arrector pili muscle of the neonatal rat on day 2 after birth as designated by arrows a in Fig. 5A and 5D. The staining became more distinct on day 7 as indicated by arrows a in Fig. 5H, 5I and 5J. A positive staining of dermal sheath for $\gamma$-SMA was observed as designated by arrows DS in Fig. 5B and 5C. The staining for $\gamma$-SMA was limited in the lower area of the hair follicle except for the bottom end (approximately the lower half of the hair bulb), which was not stained. These types of the staining were not observed during fetal stages (Figs. 2-4). The outer root sheath (ORS) as well as inner root sheath (IRS) and hair shaft (HS) were not found to be stained on day 2 or day 7 after birth (Fig. 5C). The results of immunohistochemical study regarding $\gamma$-SMA were similar to those obtained in our previous results about the localization of $\alpha$-SMA [21]. Since the strength of the immunohistochemical reaction depends on the antibody and other experimental conditions, the quantitative comparison of the two types of SMA could not be performed. The $\gamma$-SMA staining was also positive in the blood vessel and the subcutaneous muscle tissue as designated by the arrow $\mathrm{d}$ and the asterisk in Fig. 5E, respectively. The presumptive sebaceous gland cells became detectable at this stage, though they were negative for $\gamma$-SMA (PSG in Fig. 5C). The spinous and the granular layers in the epidermis were always positive (area $\mathrm{b}$ in Fig. 5J), while the basal layer showed an ambiguous or slightly positive response in the staining for $\gamma$-SMA (area $\mathrm{c}$ in Fig. 5J). As shown in a control experiment (Fig. 5F), the cornified layer of the epidermis after birth may more or less exhibit nonspecific staining. Therefore we must be careful when discussing the staining of the cornified layer in the animals after birth.

\section{Dorsal skin on day 14 after birth}

The results shown in Fig. 6A-D suggest that hair matrix cells (HM) and dermal papilla cells (DP) are largely negative in the staining for $\gamma$-CYA, though some positive cells were occasionally found. It is probably because the hair matrix and dermal papilla may contain melanocytes or vascular cells. The area between L1 and L2 contains both stained and unstained cells localized at random. As an exception, stained cells were observed frequently in the area just above the dermal papilla as shown in the circles drawn by broken lines in Fig. 6B or 6C. These $\gamma$-CYApositive cells are assumed to be the precursor cells of the hair shaft cells $[19,20]$. Above L2, the stained areas were restricted to the outer root sheath (ORS), medulla (M) and dermal sheath (DS) (Fig. 6A, 6D-G). The results suggest that $\gamma$-CYA expression ceases in response to the keratinization of the inner root sheath (IRS), and hair cortex (Cx)/ cuticle $(\mathrm{Ce})$. In contrast, the other tissues were suggested to be active as they still need $\gamma$-CYA for their functions. In general, the $\gamma$-SMA staining patterns were similar to those observed for $\gamma$-CYA (Fig. 6I-K). One difference is the staining of the medulla, which was often observed to be unstained or partly stained for $\gamma$-SMA (a: unstained in Fig. $6 \mathrm{~J}$ and $6 \mathrm{~K}$ or c: stained, b: weakly stained in Fig. $6 \mathrm{~K}$ ). This difference is more evident when analyzing cross sections. The medulla in Fig. 7C was positive for $\gamma$-CYA, while that in Fig. 7F was negative for $\gamma$-SMA. The $\gamma$-CYA staining was positive up to the upper area of the hair follicle in the medulla, outer root sheath and dermal sheath (e and $\mathrm{f}$ in Fig. $7 \mathrm{G}$ and $7 \mathrm{H}$ ). Further, some additional differences between the staining for $\gamma$-CYA and $\gamma$-SMA were observed. First; there were significant staining-positive cells for $\gamma$ CYA in the hair matrix as described earlier, while those stained for $\gamma$-SMA were scarce (compare Fig. 6B-6D with 6I, or compare Fig. 7A with 7D), and secondly; the outermost part of the upper hair bulb was positive for $\gamma$-CYA staining (a in Fig. 7A) but was negative for $\gamma$-SMA (a in Fig. 7D). The sections of Fig. 7A and 7D are adjacent. The $\gamma$-SMA positive area in the upper left hand corner in Fig. 7D displays striated muscle cells as a positive control. We currently can not provide an appropriate explanation for these differences. The pattern and/or strength of the staining for actins are altered according to the position or height of the hair follicle. In the transitional area, the stainingpositive areas may be disjoined (arrows c in Fig. 7E). The keratinized inner root sheath and hair cortex/cuticle were not stained for $\gamma$-SMA and $\gamma$-CYA (Fig. 6G, b in Fig. 7E and $7 \mathrm{~F}$ ). The expression of both $\gamma$-CYA and $\gamma$-SMA was suggested to be positive in the arrector pili muscle as shown in Fig. 7G, 7H and 7I (designated by arrows d). The staining for $\gamma$-SMA in Fig. 7I was faint in places, and possibly indicating the decay of $\gamma$-SMA. A decrease in the staining of the arrector pili muscle during the development toward adult animals was also observed for $\alpha$-SMA [21]. We speculated that neonatal rats need the arrector pili muscle to crawl around their mother without loss of body 


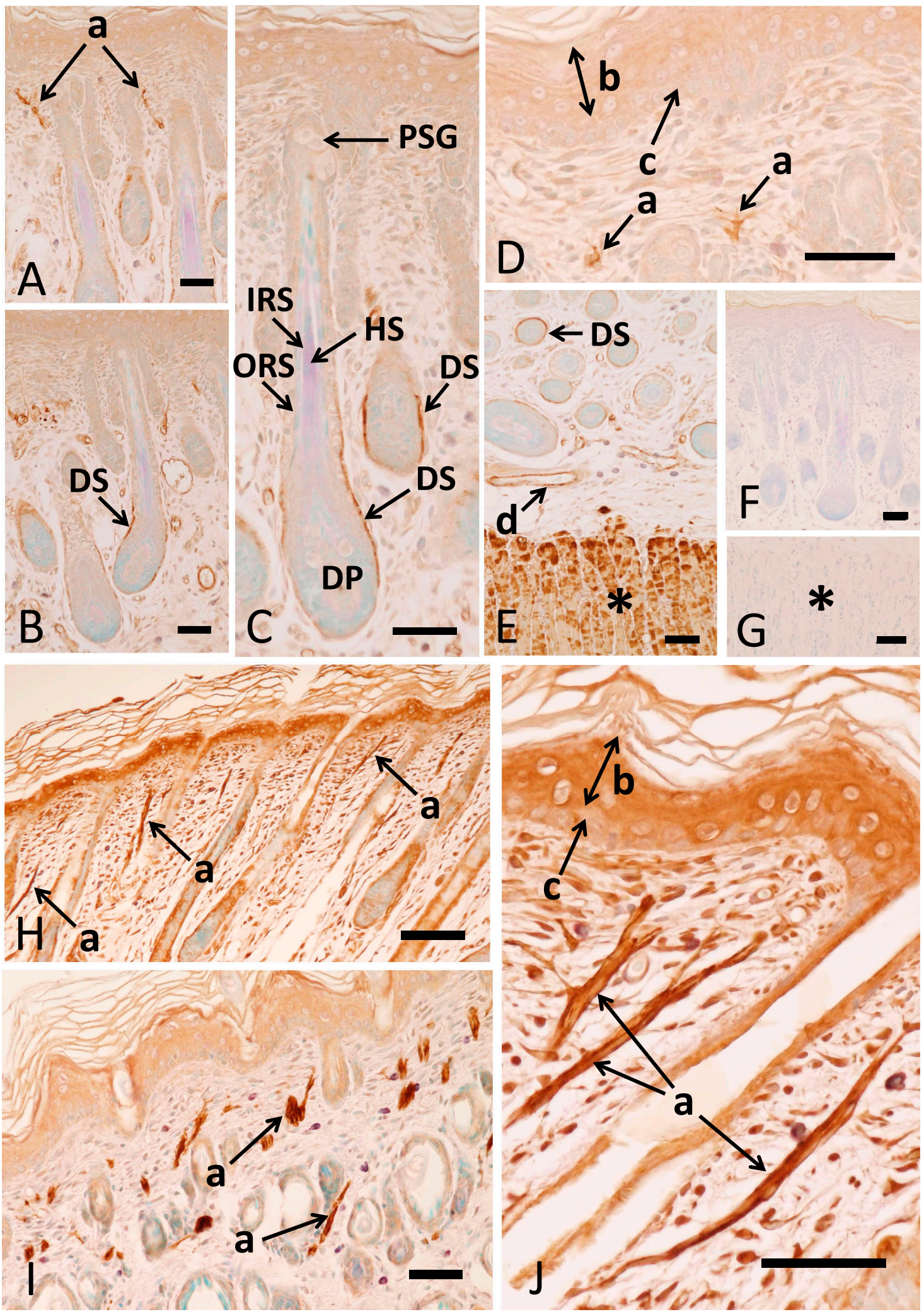

Fig. 5. Immunohistochemical staining for $\gamma$-SMA in the neonatal animals. Samples were obtained from the dorsal skin on day 2 (A-G) or on day 7 (H-J) after birth. $\mathbf{F}$ and $\mathbf{G}$ show the results of control experiments. Symbols are a: arrector pili muscle or its precursor, b: epidermis with the exception of basal layer, c: basal layer of the epidermis, d: blood vessel. The asterisk designates the subcutaneous muscle. Bars $=50 \mu \mathrm{m}(\mathbf{A}-\mathbf{G}, \mathbf{I}-\mathbf{J})$, or $100 \mu \mathrm{m}(\mathbf{H})$. 


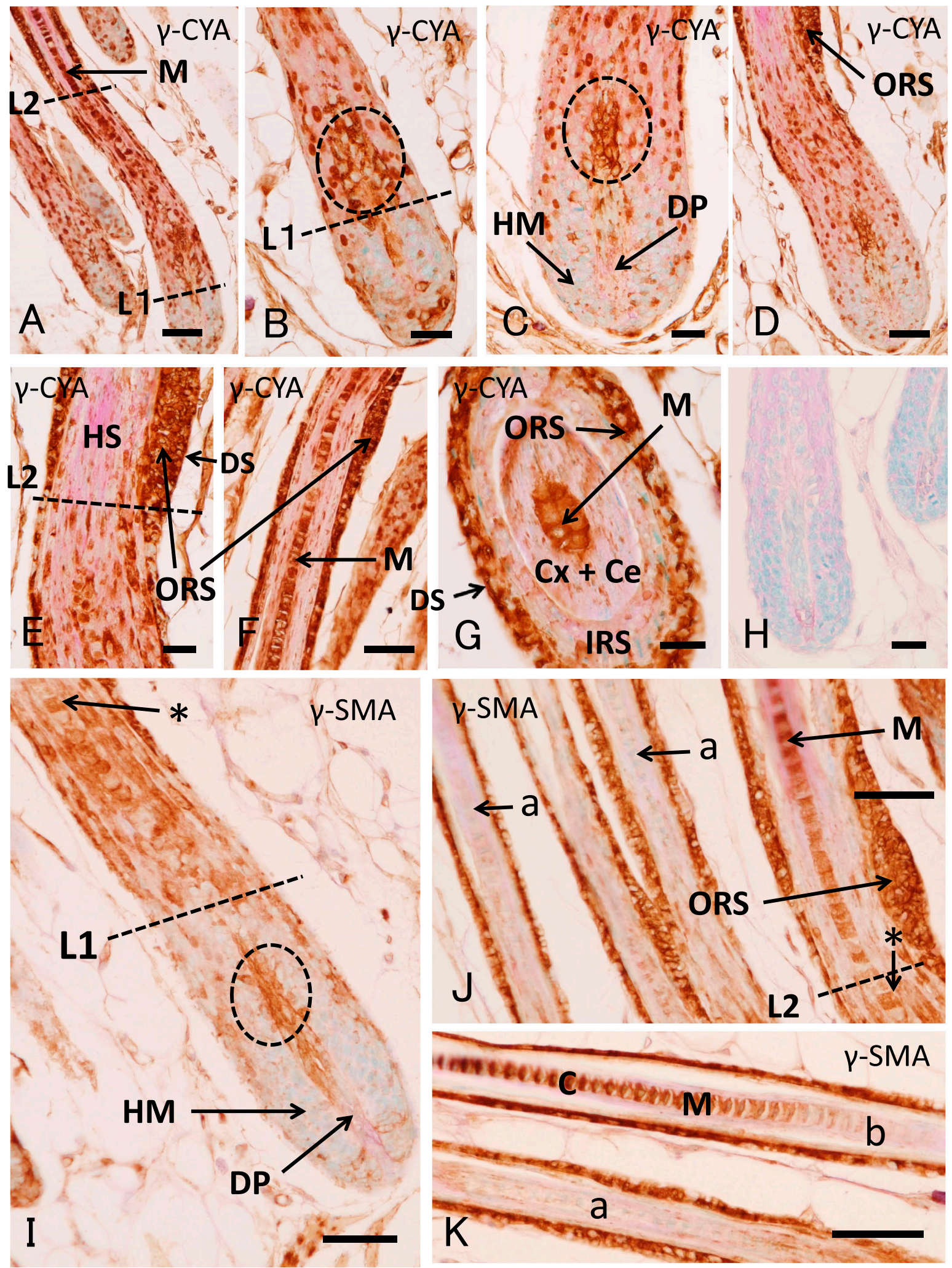

Fig. 6. Immunohistochemical staining for $\gamma$-CYA (A-G) and $\gamma$-SMA (I-K) in the dorsal skin on day 14 after birth. Panel $\mathbf{H}$ shows the result of a control experiment. Symbols are L1: a line drawn at the height of the top of dermal papilla. L2: Hair cortex, hair cuticle and inner root sheath are not stained above this line. The cells in $\mathbf{I}$ and $\mathbf{J}$ designated by asterisks are the same cell, namely panel $\mathbf{I}$ represents the lower area adjacent to panel $\mathbf{J}$. Symbols are a: unstained hair shaft, b: unstained part of the medulla, and c: stained part of the medulla. The area encircled by a broken line located just above the top of the dermal papilla in $\mathbf{B}, \mathbf{C}$ and $\mathbf{I}$ is a special where the stained cells are abundant. Bars $=50 \mu \mathrm{m}(\mathbf{A}$ and $\mathbf{I}-\mathbf{K})$ and $25 \mu \mathrm{m}(\mathbf{B}-\mathbf{H})$. 


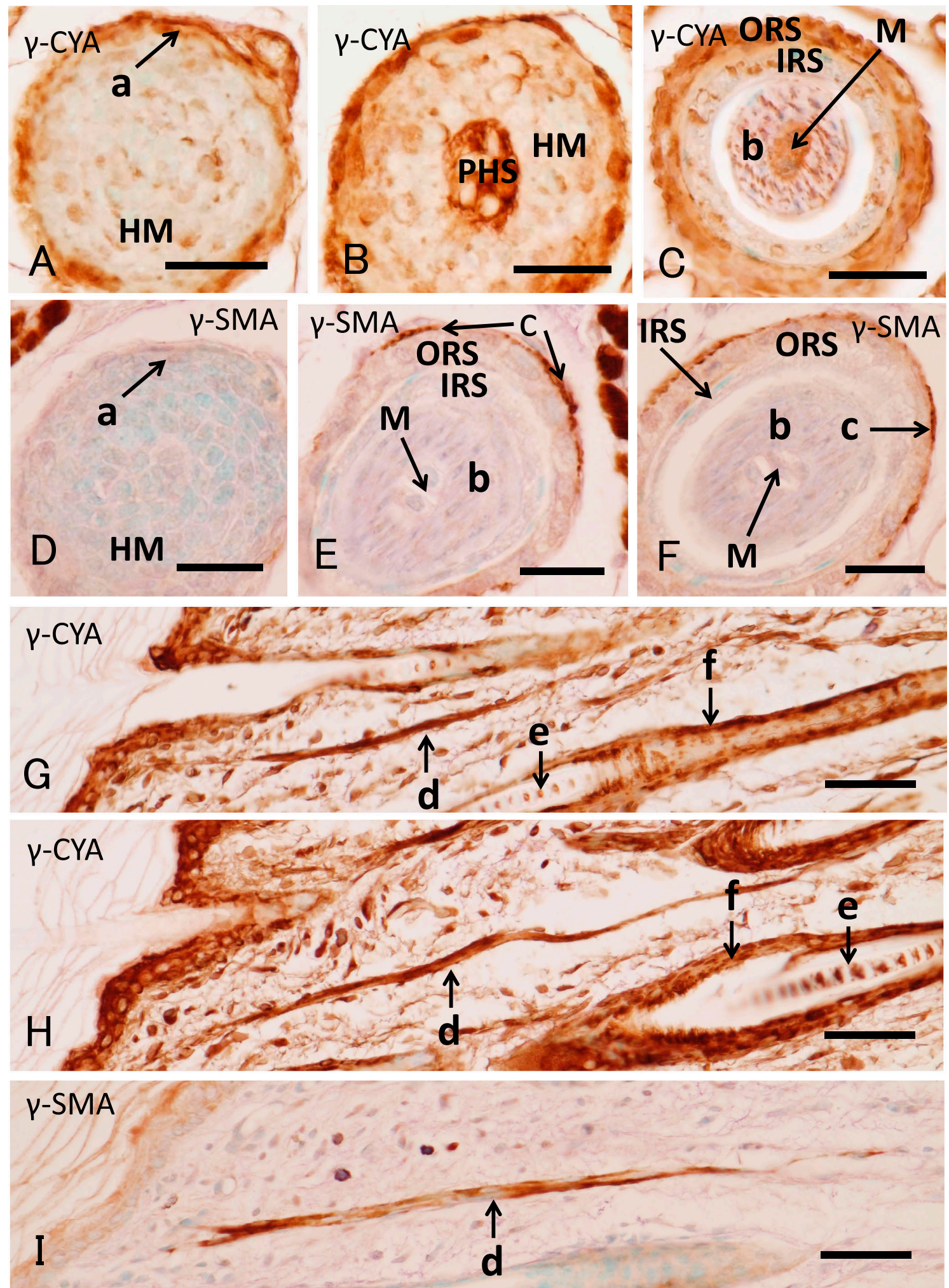

Fig. 7. Immunohistochemical staining for $\gamma$-CYA $(\mathbf{A}-\mathbf{C}, \mathbf{G}, \mathbf{H})$, and for $\gamma$-SMA $(\mathbf{D}-\mathbf{F}, \mathbf{I})$ in the dorsal skin on day 14 after birth. Symbols are a: outer root sheath and dermal sheath, b: hair cortex and hair cuticle, c: stained part of the dermal sheath, d: arrector pili muscle, e: medulla stained positive for $\gamma-$ CYA, and f: dermal sheath. PHS: precursors of the hair shaft. Bars $=25 \mu \mathrm{m}(\mathbf{A}-\mathbf{F})$ and $50 \mu \mathrm{m}(\mathbf{G}-\mathbf{I})$. 
temperature, and thereafter, the necessity decreases as the animals grow and their sizes increase.

\section{Mystacial pad on day 14 after birth}

Not later than one or two weeks after birth, the typical structure of vibrissa is accomplished in the mystacial pad of the rat. That is, vibrissal capsule, vibrissal shaft, ring sinus, cavernous sinus, root sheaths, dermal sheath, dermal papilla, sebaceous gland, vibrissal nerve, ringwulst and other accessory tissues are formed and located at their positions in the vibrissal follicle. Similar to the pelage hair, a majority of the dermal papilla cells as well as the hair matrix cells of the vibrissae were negative in the staining for $\gamma$ CYA (Fig. 8A). In contrast, the vibrissal capsule surrounding the follicle was positive (VC in Fig. 8A). The oblique section shows that some stained hair matrix cells locate in the upper half of the hair matrix being adjacent to the dermal papilla (Fig. 8B). One of them, positive for $\gamma$-CYA, is designated by the asterisk in Fig. 8B. This area designated by " $\mathrm{a}$ " is known to be a zone containing melanocytes [2]. The zone designated by " $b$ " in Fig. $8 \mathrm{~B}$ is assumed to contain precursors of the hair shaft [20]. Figs. 8A and 7B show that the lower part of the hair matrix (HM) was not stained, while significant numbers of cells in the upper part were stained. The zone "c", which is assumed to be the precursor of the Huxley's layer, was not stained, though the zone "d", which is assumed to be the precursor of the Henle's layer was stained. The cells of the Huxley's layer (Hx) became positive when they proceeded to the upper area (Fig. 8C). Since the differentiation starts earlier in the Henle's layer (He) than that in the Huxley's layer [20], the $\gamma$-CYApositive stage may be observed earlier in the Henle's layer (Fig. 8B). An oblique section at the position between the lower and middle height of the vibrissa is shown in Fig. 8C and $8 \mathrm{D}$ (the center part of $8 \mathrm{D}$ is enlarged in $8 \mathrm{C}$ ). At this height, the outer root sheath (ORS) and the Huxley's layer (Hx) are clearly positive, while the staining of the Henle's layer becomes ambiguous. When the Henle's layer is highly keratinized, the staining may decrease (Fig. 8C). The staining of the hair cortex $(\mathrm{Cx})$ was weak but detectable, while that of the hair cuticle $(\mathrm{Ce})$ was negative at this height (Fig. 8C). In the upper area of the hair shaft, both $\mathrm{Cx}$ and Ce were negative in the staining for $\gamma$-CYA (Fig. 8G) probably due to the progress of the keratinization.

Fig. 8D shows that the trabeculae $(\mathrm{Tb})$ in the ring sinus (RS), vibrissal capsule (VC) and vibrissal nerve (VN) were positive in the staining for $\gamma$-CYA. Fig. 8E shows a layer designated as "e" located at the outermost area of the outer root sheath. The cells in the "e" layer were stained for $\gamma$-CYA in their distal side facing the basement membrane (BM) indicating that these cells present an intracellular polarity for the staining. The $\gamma$-CYA staining in the vibrissal capsule is compared with that of the attached striated muscle in Fig. 8F. It shows that most vibrissal capsule cells were stained, while the majority of the striated muscle cells (STM) were not stained or stained very weakly. The expres- sion of $\gamma$-CYA in the inner root sheath seemed to decrease during the cornification of these tissues (Fig. 8G). The left side of Fig. $8 \mathrm{G}$ is equivalent to the upper side. The inner root sheath $\gamma$-CYA positive cells are seen in the right side of Fig. $8 \mathrm{G}$.

Fig. 9A suggests that $\gamma$-SMA slightly expressed in the upper half of the hair bulb designated by a. It may be detectable when compared with the hair matrix (HM) in the lower half or the control experiment shown in Fig. 9B. However, the expression level was very low in comparison to that in the striated muscle designated by arrow $b$ in Fig. 9A, which is shown as a positive control. In the middle height of the vibrissa, $\gamma$-SMA was suggested to be localized at the dermal sheath (DS) as shown in Fig. 9D-9F, and 9I, though the staining was not observed in a lower section (Fig. 9C). The staining of outer root sheath was weak (Fig. 9D-9G, and 9I), but not negative depending on its height (for example, ORS in Fig. 9G). In contrast to the case of $\gamma$ CYA, the staining for $\gamma$-SMA was strong in the striated muscle and was weak in the vibrissal capsule (Fig. 9D and 9H). In Fig. 9I, the medulla was not stained for $\gamma$-SMA. However, it should be noted that stained medulla could be found (Fig. 6K). The dermal striated muscle was positive in the staining for $\gamma$-SMA, and Fig. 9J-9L shows that the staining for $\gamma$-SMA itself formed striation, suggesting that $\gamma$-SMA is located in a special band of the voluntary muscle. The vibrissal capsule and the adjacent striated muscle were entangled with each other, though the occurrence was occasionally (arrows c in Fig. 9H). Trabeculae in the cavernous sinus and ringwulst were stained weakly (Fig. 9G and 9H). Erythrocytes in the ring or cavernous sinus were not stained (Fig. 9G and 9H).

\section{Mystacial pad on day 61 after birth}

Fig. 10A shows the positive staining for $\gamma$-SMA designated by arrow a in the dermal sheath. The positive area starts from the middle height of the hair bulb and extended to positions higher than the hair bulb. Fig. 10A also shows a positive staining of the outer root sheath (ORS). The staining of the dermal sheath presented an upper limit (dotted line) in the middle height of the vibrissal follicle as shown in Fig. 10B. Hair shaft, inner root sheath and outer root sheath were not stained at this height (Fig. 10B). Fig. $10 \mathrm{C}-\mathrm{E}$ are views of cross sections cut at the middle height position of the hair bulb (Fig. 10C), at high position of the hair bulb (Fig. 10D), and at the position just above the hair bulb (Fig. 10E), respectively. In all these sections, dermal sheath was shown to be positive in the staining for $\gamma$-SMA (arrow a). Fig. 10F is a cross section cut at the position of low height of the vibrissal follicle, although it is higher than the position of Fig. 10E. It shows the staining for $\gamma$ SMA in the dermal sheath as designated by arrow a. The boundary between outer root sheath and inner root sheath may be slightly positive as designated by an arrow c. Fig. $10 \mathrm{G}$ shows a large vibrissa stained for $\gamma$-SMA. The part surrounded by the dotted rectangle in Fig. 10G is enlarged 


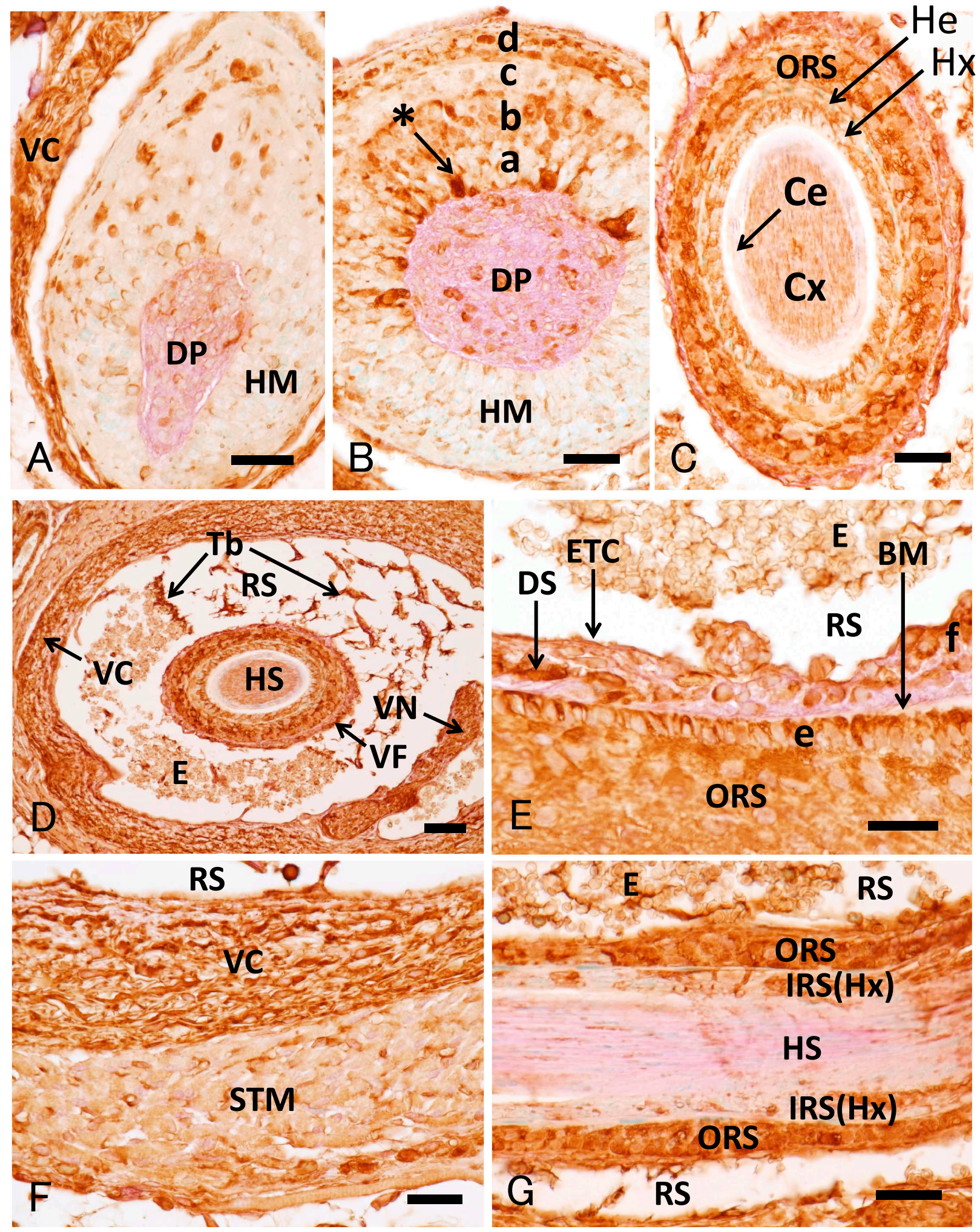

Fig. 8. Immunohistochemical staining for $\gamma$-CYA in the vibrissae on day 14 after birth. Symbols a-d represent the layers designated tentatively from inside to outside in the upper area of the hair bulb. Other symbols are e: outermost cellular monolayer in the outer root sheath, and f: a root area of the ringwulst. The asterisk in $\mathbf{B}$ designates the stained cell adjacent to the dermal papilla. Bars $=25 \mu \mathrm{m}$ (except $\mathbf{D})$ and $50 \mu \mathrm{m}(\mathbf{D})$ 


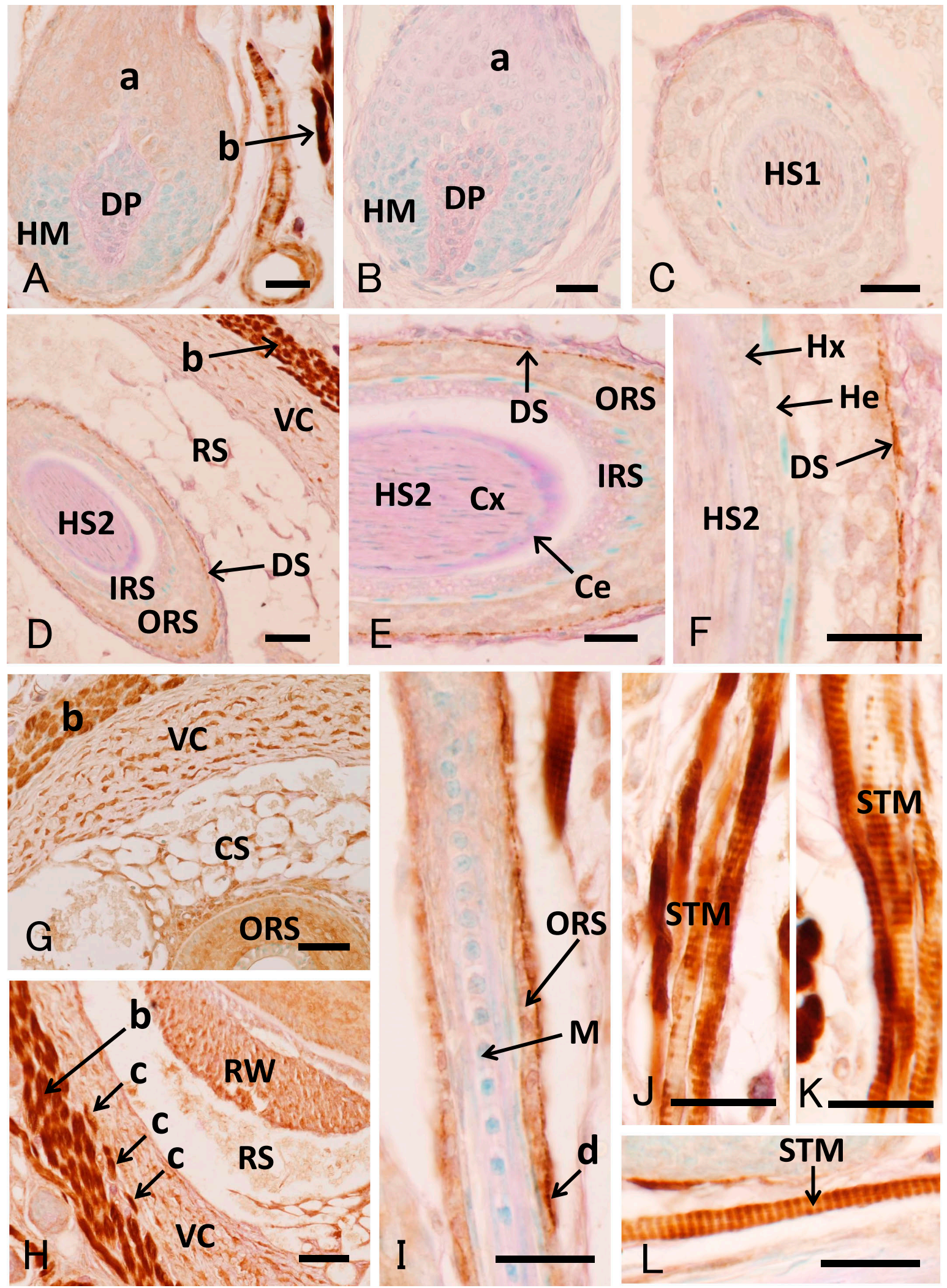

Fig. 9. Immunohistochemical staining for $\gamma$-SMA in the vibrissae on day 14 after birth. The result of a control experiment is shown in B. Symbols are a: basal part of the hair shaft, b: striated muscle, c: muscle cells in the vibrissal capsule, and d: the stained area of dermal sheath. HS1 and HS2 are different sections of the hair shaft. The level of the keratinization is HS1<HS2. The position of HS2 is higher than that of HS1. Bars $=25 \mu \mathrm{m}(\mathbf{A}-\mathbf{C}, \mathbf{E}, \mathbf{F}, \mathbf{I}, \mathbf{L})$ and $50 \mu \mathrm{m}(\mathbf{D}, \mathbf{G}$ and $\mathbf{H})$. 


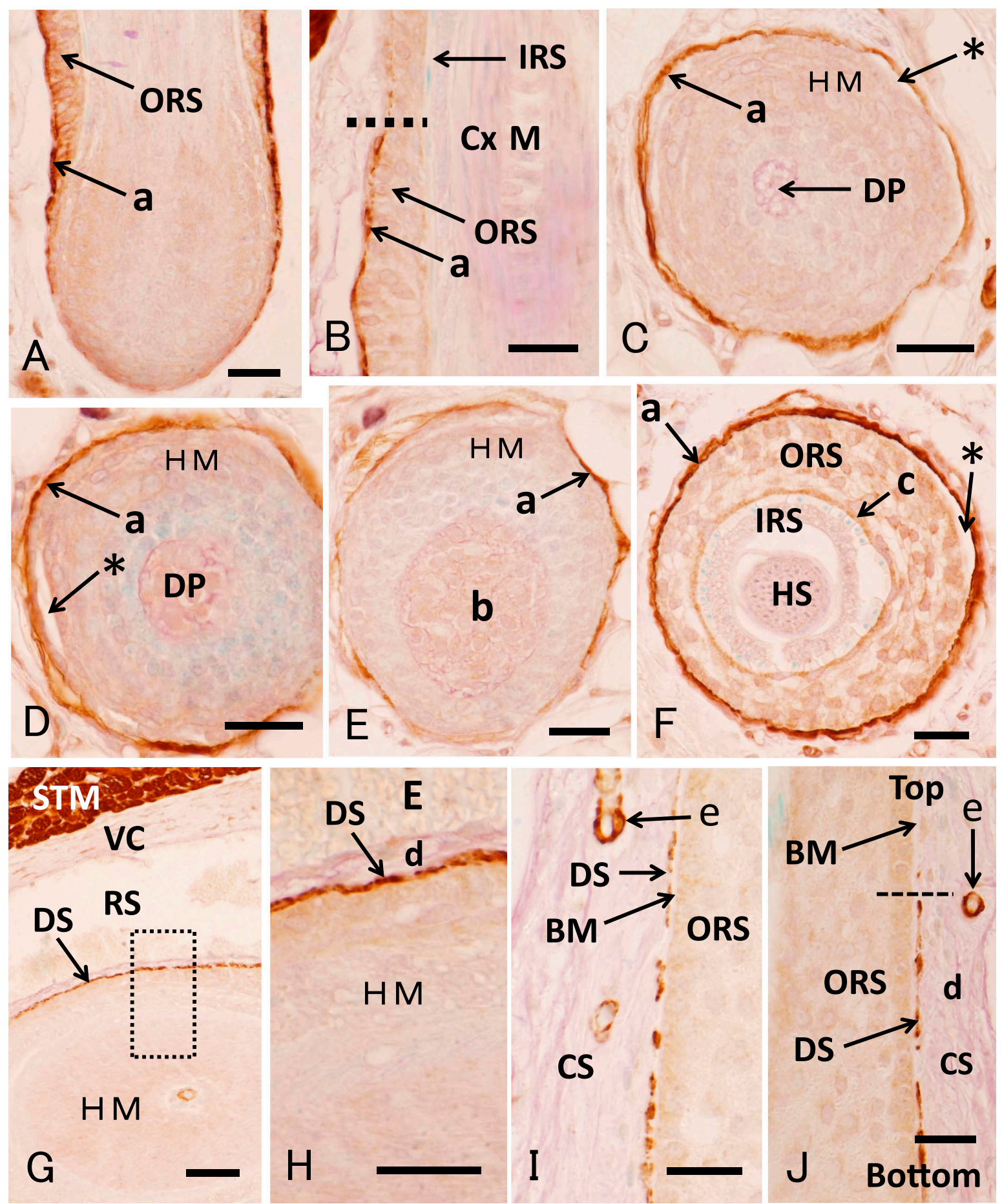

Fig. 10. Immunohistochemical staining for $\gamma$-SMA in the vibrissal follicle on day 61 after birth. Symbols are a: the stained area of dermal sheath, b: basal part of the hair shaft, c: stained area located at the boundary of outer and inner root sheaths, d: undetermined cells between dermal sheath and sinus, and e: stained cells in the cavernous sinus. A box enclosed by dotted lines is enlarged and shown in $\mathbf{H}$. The gaps designated by asterisks in $\mathbf{C}, \mathbf{D}$, and $\mathbf{F}$ may be the results of mechanical stress at the basement membrane-equivalent boundary between the dermis and the epidermis during the operation of the sectioning. A broken line in $\mathbf{J}$ represents is the boundary between stained and unstained areas of dermal sheath. Bars $=25 \mu \mathrm{m}$.

and shown in Fig. 10H. It presents a positive staining for $\gamma$ SMA distinctly in the dermal sheath. It also shows the unstained cellular layers (d) in the distal side of the dermal sheath. The layer adjacent to the sinus (E filled with erythrocytes) may consist of endothelial cells. However, the layer observed between the endothelium and dermal sheath has not been studied so far. We can detect an unstained canal between the dermis and epidermis in Fig. 10I or 10J designated as BM. This confirms that the distinct stained area is the dermal sheath, because BM is thought to be 
equivalent to the basement membrane. The staining of the dermal sheath was disjoined in the middle part of the follicle (Fig. 10I) and faded out in the area above the broken line (Fig. 10J). This pattern of the staining resembles closely with that of staining for $\alpha$-SMA described previously [21]. Although the cells between the dermal sheath and the sinus ( $\mathrm{d}$ in Fig. 10J) were unstained, the cells that seemed to form a blood vessel (designated as e) in the area $\mathrm{d}$ were positive for $\gamma$-SMA.

Ringwulst (RW), sinus-related tissues, and sebaceous gland (SG) located in the upper area of the vibrissal follicle were studied in specimens from animals of day 61 after birth (Fig. 11). The ringwulst (RW), trabeculae (a tissue like a net in CS), vibrissal capsule (VC), and outer root sheath (ORS) were stained more or less for $\gamma$-CYA (Fig. 11A), while these tissues were scarcely stained for $\gamma$-SMA (Fig. 11B). A distinct stain for $\gamma$-SMA was observed only in the striated muscle (STM) to move vibrissa in the vicinity of ringwulst (RW) as shown in Fig. 11B. In the upper area of the follicle (Fig. 11B), the staining for $\gamma$-SMA was not observed in the dermal sheath. In Fig. 11C, the cells stained for $\gamma$-CYA were observed between the outer root sheath and ring sinus. Endothelial cells, dermal sheath cells, and other uncharacterized cells are assumed to be located in this area. Outer root sheath (ORS) cells, especially the cells located at the distal end were stained conspicuously (Fig. 11C). The outermost area (arrow a) and the innermost area (arrow b) of the inner root sheath (IRS) were not stained (Fig. 11C). The former is thought to be the Henle's layer and the latter to be the cuticle of inner root sheath, according to their locations. These layers are known to keratinize earlier than the Huxley's layer designated as "c" in Fig. 11C that lies in the center part of the inner root sheath [20]. The Huxley's layer showed a distinct staining for $\gamma$-CYA even at this high position of the follicle (Fig. 11C). As shown in Fig. 11D$11 \mathrm{~F}$, sebaceous glands (SG) were largely positive in the staining of $\gamma$-CYA. This staining consists of a number of stained particles in the cytoplasm, and such pattern is specific for the sebaceous gland. It is known that the sebaceous gland performs holocrine secretions [34], but our results did not show a staining gradient according to the line from new (upper area of SG) to old (lower or near cavity area of SG) cells. The cavity between the hair shaft and the sheath is designated as a symbol $\mathrm{d}$ in Fig. 11F. The pattern of the expression of $\gamma$-SMA was more diverse (Fig. 11G-11I), that is, negative (Fig. 11G), positive (Fig. 11H), and mixed type (Fig. 11I) of sebaceous glands were found. It suggests that the expression may depend on the situation of the gland including the on/off switch of the secretion.

\section{Discussion}

The pilosebaceous unit is a mammal-specific organ and is composed of hair follicle, arrector pili involuntary muscle, and sebaceous gland. The major roles of this organ are to keep warmth, to mitigate impact, and to shade sun- light. In addition to the pelage hair, most tetrapods have vibrissae and are used as sensors. The vibrissa contains vibrissal capsule and blood sinus that surrounds the basic pelage-type hair structure. Furthermore it is accompanied by vibrissal nerve fibers, voluntary muscles, and other accessory tissues. Their physiological functions remain unknown. However, it is not accompanied by the arrector pili muscle $[5,13,22]$. The hair follicle has a minute structure, which consists of a central hair shaft and surrounding multilayers including three layers of inner root sheath, outer root sheath consisting of several types of the cells, the basement membrane and the dermal sheath $[9,19,20]$. The role of these surrounding multilayers has been one of the major interest for hair investigators. We studied the possibility that some areas of dermal sheath and outer root sheath may play a role as a smooth muscle $[21,22]$. In addition to the $\alpha$-SMA $[10,21]$, we found that $\gamma$-SMA was also localized in the lower area of the dermal sheath and outer root sheath, suggesting that these tissues function like a smooth muscle. Since the dermal sheath is usually a monolayer tissue presenting no blood vessels, it is unlikely that the cells expressing SMA function as a component of the blood vessels. It should be noted that the area where SMA expression is high, seems to be squeezed, in contrast to the hair bulb that seems to be expanded spherically as the cells proliferate. This power of squeezing may be yielded by the SMA in dermal sheath or outer root sheath. The shape and nature of the hair follicle are not only determined by an external power, but are also thought to be controlled by asymmetric cell division or other regulatory systems of proliferation and differentiation [31]. The compliance that is suggested to be yielded by $\gamma$-SMA [1] may be beneficial for the maintenance of hair follicles against the deformation forced by the external contact or the internal muscle activity.

The localization of $\gamma$-SMA in the arrector pili muscle suggested its participation in the contraction of this muscle together with $\alpha$-SMA [21]. The staining for $\gamma$-CYA was also positive in the arrector pili muscle. The expression of $\gamma$-CYA may support the cell movement and the extension of cell shape needed for the differentiation of the arrector pili muscle. It is intriguing that besides the expression of $\gamma$ CYA and $\gamma$-SMA determined in this study, $\alpha$-SMA [21], pan-actin, and nonmuscle myosins [22] were suggested to be scarce in the skin basal layer, hair bud, and hair matrix. The function of these tissues to provide new cells, suggesting that a very small amount of actin and myosin is required for cell proliferation. In contrast, the granular and spinous layers expressed the above mentioned proteins (this study and refs. 21 and 22), suggesting that a large amount and various types of actin and myosin are produced for the differentiation of epidermal cells. The staining of sebaceous glands for $\gamma$-CYA was largely positive, but the strength was variable from one cell to another and from one organ to another. The staining for $\gamma$-SMA was more variable. Some cells or organs appeared to be almost negative. This variability is not due to the instability of the antibody, because 


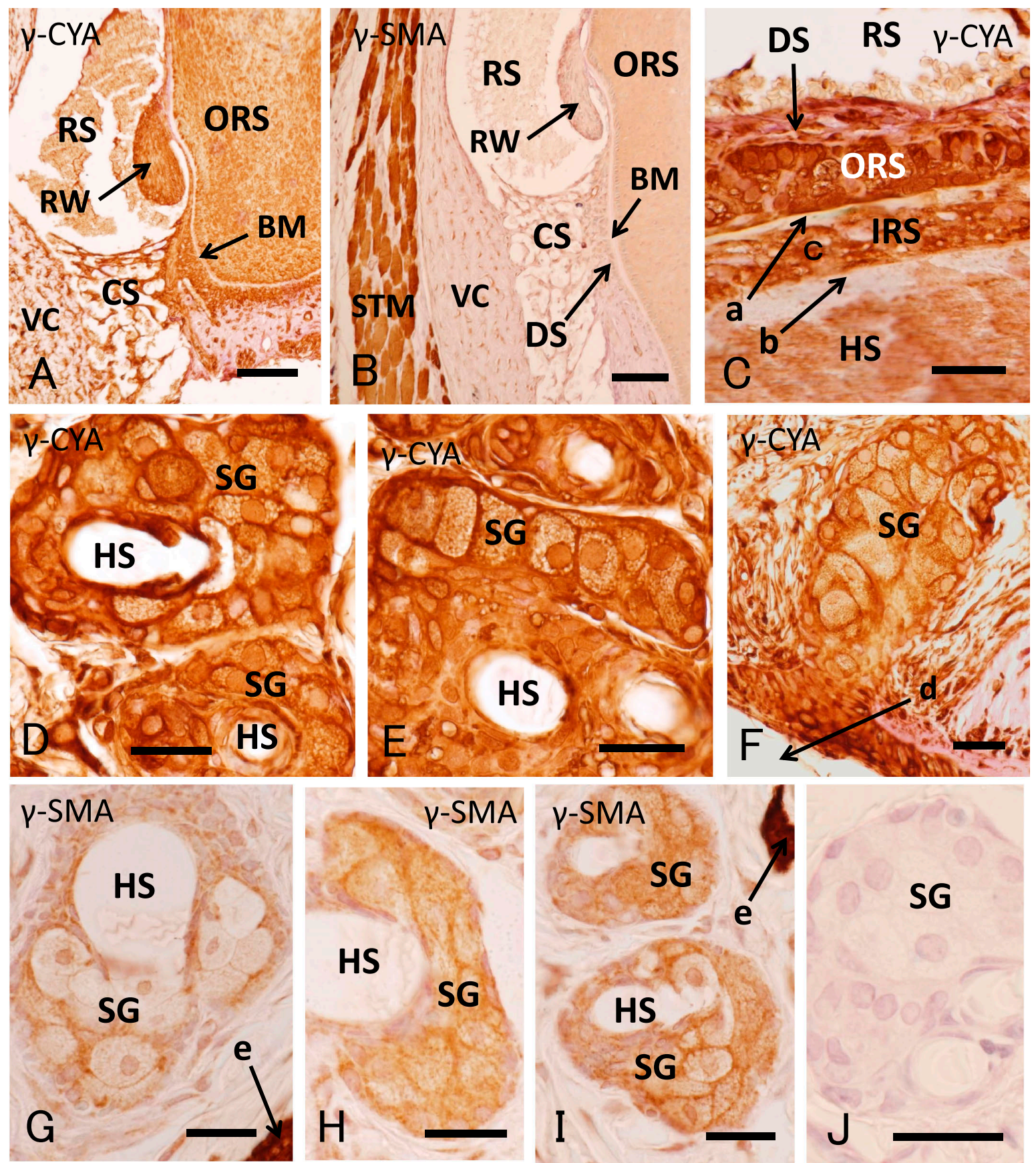

Fig. 11. Immunohistochemical staining for $\gamma$-actins in the vibrissal follicle on day 61 after birth with specific reference to sinus and sebaceous gland. A, C-F show the results of the staining for $\gamma$-CYA and $\mathbf{B}, \mathbf{G}-\mathbf{I}$ for $\gamma$-SMA. The results of a control experiment is shown in J. Symbols a: Henle's layer, b: cuticle of inner root sheath, c: Huxley's layer, d: a pore (crevice) of the skin formed between outer root sheath and inner root sheath, and e: muscle as a positive control. Bars $=100 \mu \mathrm{m}(\mathbf{A}, \mathbf{B})$ and $25 \mu \mathrm{m}(\mathbf{C}-\mathbf{I})$.

the adjacent muscles were steadily stained. We speculate that the activity of the sebaceous gland may be controlled by some signals issued on demand and that these signals influence the expression of $\gamma$-actins.

In the embryo, $\gamma$-CYA expression was clearly observed in the embryonic skin (Fig. 1). This staining did not seem to be associated with the keratinization. Several cellular layers, piled up beneath the periderm, exhibited a highly positive staining except for the hair bud and the partial area of the deepest epidermal layer. Taking the staining of all other areas into account, we speculate that the standard program of the expression of $\gamma$-CYA in the cells is "on" in the embryo, and that the negative or lightly stained cells must be involved in the functions that do not require $\gamma$ CYA, such as emphasizing proliferation in the hair bud, abrogation of motility in erythrocytes, and special differentiation in the striated muscle. Since terminally differentiated tissues increased in the adult, $\gamma$-CYA negative tissues 
became more common. Typically, terminally differentiated hair cortex and hair cuticle have scarce immunohistochemical response to the antibody. In contrast to the expression of $\gamma$-CYA, that of $\gamma$-SMA may be usually "off" in both embryonic and adult tissues, and the positive cells must be involved in the functions that require $\gamma$-SMA. In this study, we showed some areas expressing $\gamma$-SMA. Among them, the striated muscle in the mystacial pad is interesting. The staining pattern for $\gamma$-SMA formed stripes in the muscle, but their shades diverged from one area to another, suggesting that the filaments are composed of a mix of the isoforms and the ratio of $\gamma$-SMA is not fixed, as Perrin and Ervasti used the term "alloy" as a metaphor for actin filaments [27].

\section{Abbreviations}

$\mathrm{BM}$; basement membrane, BL; basal layer of the epidermis, Ce; hair cuticle, $\mathrm{Cx}$; hair cortex, CS; cavernous sinus, DP; dermal papilla, DS; dermal sheath, E; erythrocyte, ETC; endothelial cell, HB; hair bud, He; Henle's layer, Hx; Huxley's layer, HM; hair matrix, HS; hair shaft, IRS; inner root sheath, M; medulla, ORS; outer root sheath, PBM; presumptive basement membrane, Pd; periderm, PHF; primitive hair follicle, PSG; presumptive sebaceous gland cells, RS; ring sinus, RW; ringwulst, SG; sebaceous gland, STM; striated muscle, Tb; trabecula, VB; vibrissal bud, VC; vibrissal capsule, VF; vibrissal follicle, VN; vibrissal nerve.

\section{Acknowledgement}

We are grateful to Dr. Yukihisa Hamaguchi for his support and advice.

\section{References}

1. Arnoldi, R., Hiltbrunner, A., Dugina, V., Tille, J-C. and Chaponnier, C. (2013) Smooth muscle actin isoforms: A tug of war between contraction and compliance. Eur. J. Cell Biol. 92; 187-200.

2. Birbeck, M. S. C. and Mercer, E. H. (1956) The electron microscopy of the human hair follicle. I. Introduction and the hair cortex. J. Biophys. Biochem. Cytol. 3; 203-214.

3. Bunnell, T. M. and Evrasti, J. M. (2010) Delayed embryonic development and impaired cell growth and survival in Actg1 null mice. Cytoskeleton 67; 564-572.

4. Dugina, V., Zwaenepoel, I., Gabbian, G., Clement, S. and Chaponnier, C. (2009) $\beta$ - and $\gamma$-cytoplasmic actins display distinct distribution and functional diversity. J. Cell Sci. 122; 2980-2988.

5. Ebara, S., Kumamoto, K., Matsuura, T., Mazurkiewicz, J. E. and Rice, F. L. (2002) Similarities and differences in the innervation of mystacial vibrissal follicle-sinus complexes in the rat and cat: A confocal microscopic study. J. Comp. Neurol. 449; 103-119.

6. Goodson, H. V. and Hawse, W. F. (2002) Molecular evolution of the actin family. J. Cell Sci. 115; 2619-2622.

7. Haidarliu, S., Simony, E., Golomb, D. and Ahissar, E. (2010) Muscle architecture in the mystacial pad of the rat. Anat. Rec. $293 ; 1192-1206$.
8. Hanft, L. M., Rybakova, I. N., Patel, J. R., Rafael-Forney, J. A. and Ervasti, J. M. (2006) Cytoplasmic gamma-actin contributes to a compensatory remodeling response in dystrophin-deficient muscle. Proc. Natl. Acad. Sci. U S A 103; 5385-5390.

9. Hardy, M. H. (1992) The secret life of the hair follicle. Trends Genet. 8; 55-61.

10. Jahoda, C. A. B., Reynolds, A. J., Chaponnier, C., Forester, J. C. and Gabbiani, G. (1991) Smooth muscle $\alpha$-actin is a marker for hair follicle dermis in vivo and in vitro. J. Cell Sci. 99; 627-636.

11. Jockusch, B. M. and Graumann, P. L. (2011) The long journey: Actin on the road to pro- and eukaryotic cells. Rev. Physiol. Biochem. Pharmacol. 161; 67-85.

12. Khaitlina, S. Y. (2001) Functional specificity of actin isoforms. Int. Rev. Cytol. 202; 35-98.

13. Kim, J-N., Koh, K-S., Lee, E., Park, S-C. and Song, W-C. (2011) The morphology of the rat vibrissal follicle-sinus complex revealed by three-dimensional computer-aided reconstruction. Cells Tissues Organs 193; 207-214.

14. Klar, J., Raykova, D., Gustafson, E., Tothova, I., Ameur, A., Wanders, A. and Dahl, N. (2015) Phenotypic expansion of visceral myopathy associated with ACTG2 tandem base substitution. Eur. J. Hum. Genet. doi:10.1038/ejhg.2015.49.

15. Kumar, A., Crawford, K., Close, L., Madison, M, Lorenz, J., Doetschman, T., Pawlowski, S., Duffy, J., Neumann, J., Boivin, G. P., O'Toole, B. A. and Lessard, J. L. (1997) Rescue of cardiac $\alpha$-actin-deficient mice by enteric smooth muscle $\gamma$-actin. Proc. Natl. Acad. Sci. U S A 94; 4406-4411.

16. M'Boneko, V. and Merker, H. J. (1988) Development and morphology of the periderm of mouse embryos (days 9-12 of gestation). Acta Anat. (Basel). 133; 325-336.

17. McHugh, K. M., Crawford, K. and Lessard, J. L. (1991) A comprehensive analysis of the developmental and tissue-specific expression of the isoactin multigene family in the rat. Develop. Biol. 148; 442-458.

18. Miwa, T., Manabe, Y., Kurokawa, K., Kamada, S., Kanda, N., Bruns, G., Ueyama, H. and Kakunaga, T. (1991) Structure, chromosomal location, and expression of the human smooth muscle (enteric type) $\gamma$-actin gene: Evolution of six human actin genes. Mol. Cell. Biol. 11; 3296-3306.

19. Morioka, K. (2009) A guide to hair follicle analysis by transmission electron microscopy: technique and practice. Exp. Dermatol. 18; 577-582.

20. Morioka, K. (2005) Hair Follicle: Differentiation under Electron Microscope. An Atlas. Springer Verlag, Tokyo.

21. Morioka, K., Arai, M. and Ihara, S. (2011) Steady and temporary expressions of smooth muscle actin in hair, vibrissa, arrector pili muscle, and other hair appendages of developing rats. Acta Histochem. Cytochem. 44; 141-153.

22. Morioka, K., Matsuzaki, T. and Takata, K. (2006) Localization of myosin and actin in the pelage and whisker hair follicles of rat. Acta Histochem. Cytochem. 39; 113-123.

23. Mounier, N., Perriard, J.-C., Gabbiani, G. and Chaponnier, C. (1997) Transfected muscle and non-muscle actins are differentially sorted by cultured smooth muscle and non-muscle cells. J. Cell Sci. 110; 839-846.

24. Munjal, A. and Lecuit, T. (2014) Actomyosin networks and tissue morphogenesis. Development 141; 1789-1793.

25. Nakata, T., Nishina, Y. and Yorifuji, H. (2001) Cytoplasmic $\gamma$ actin as a Z-disc protein. Biochem. Biophys. Res. Commun. 286; 156-163.

26. Papponen, H., Kaisto, T., Leinonen, M. and Metsikko, K. (2009) Evidence for gamma-actin as a $\mathrm{Z}$ disc component in skeletal myofibers. Exp. Cell Res. 315; 218-225.

27. Perrin, B. J. and Ervasti, J. M. (2010) The actin gene family: Function follows isoform. Cytoskeleton 67; 630-634.

28. Pinder, J. C. and Gratzer, W. B. (1983) Structural and dynamic 
states of actin in the erythrocyte. J. Cell Biol. 96; 768-775.

29. Pollard, T. D. and Cooper, J. A. (2009) Actin, a central player in cell shape and movement. Science 326; 1208-1212.

30. Richardson, R. J., Hammond, N. L., Coulombe, P. A., Saloranta, C., Nousiainen, H. O., Salonen, R., Berry, A., Neil Hanley, N., Headon, D., Karikoski, R. and Dixon, M. J. (2014) Periderm prevents pathological epithelial adhesions during embryogenesis. J. Clin. Invest. 124; 3891-3900.

31. Roubinet, C. and Cabernard, C. (2014) Control of asymmetric cell division. Curr. Opin. Cell Biol. 31; 84-91.

32. Schleicher, M. and Jockusch, B. M. (2008) Actin: its cumbersome pilgrimage through cellular compartments. Histochem. Cell Biol. 129; 695-704.

33. Takano-Ohmuro, H., Mukaida, M. and Morioka, K. (1996) Distribution of actin, myosin, and spectrin during enucleation in erythroid cells of hamster embryo. Cell Motil. Cytoskeleton 34; 95-107.

34. Thody, A. J. and Shuster, S. (1989) Control and function of sebaceous glands. Physiol. Rev. 69; 383-416.

35. Tondeleir, D., Lambrechts, A., Muller, M., Jonckheere, V., Doll, T., Vandamme, D., Bakkali, K., Watershoot, D., Lemaistre, M., Debeir, O., Decaestecker, C., Hinz, B., Staes, A., Timmerman,
E., Colaert, N., Gevaert, K., Vandekerckhove, J. and Ampe, C. (2012) Cells lacking $\beta$-actin are genetically reprogrammed and maintain conditional migratory capacity. Mol. Cell Proteomics. $11 ; 255-271$.

36. Tondeleir, D., Vandamme, D., Vandekerckhove, J., Ampe, C. and Lambrechts, A. (2009) Actin isoform expression patterns during mammalian development and in pathology: Insights from mouse models. Cell Motil. Cytoskeleton 66; 798-815.

37. Vandekerckhove, J. and Weber, K. (1978) At least six different actins are expressed in a higher mammal: An analysis based on the amino acid sequence of the amino-terminal tryptic peptide. $J$. Mol. Biol. 126; 783-802.

38. Zhu, M., Yang, T., Wei, S., DeWan, A. T., Morell, R. J., Elfenbein, J. L., Fisher, R. A., Leal, S. M., Smith, R. J. and Friderici, K. H. (2003) Mutations in the gamma-actin gene (ACTG1) are associated with dominant progressive deafness (DFNA20/26). Am. J. Human Genet. 73; 1082-1091.

This is an open access article distributed under the Creative Commons Attribution License, which permits unrestricted use, distribution, and reproduction in any medium, provided the original work is properly cited. 